\title{
Effect and Molecular Mechanisms of Jiedu Recipe on Hypoxia-Induced Angiogenesis after Transcatheter Arterial Chemoembolization in Hepatocellular Carcinoma
}

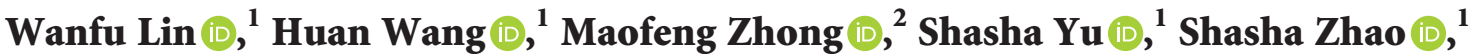 \\ Shufang Liang $\mathbb{D}$, , Juan Du $\mathbb{D}$, ${ }^{1}$ Binbin Cheng $\mathbb{D}^{1},{ }^{1}$ Wei $G u \mathbb{D}^{1},{ }^{1}$ and Changquan Ling $\mathbb{D}^{1}$ \\ ${ }^{1}$ Department of Traditional Chinese Medicine, Changhai Hospital, Second Military Medical University, Shanghai 200433, China \\ ${ }^{2}$ Department of Gastroenterology, Shanghai Municipal Hospital of Traditional Chinese Medicine Affiliated to \\ Shanghai University of TCM, Shanghai 201900, China
}

Correspondence should be addressed to Binbin Cheng; cbb8202@126.com, Wei Gu; sam6116@163.com, and Changquan Ling; changquanling@smmu.edu.cn

Received 5 January 2020; Revised 23 July 2020; Accepted 30 December 2020; Published 12 January 2021

Academic Editor: Muhammad Nabeel Ghayur

Copyright (c) 2021 Wanfu Lin et al. This is an open access article distributed under the Creative Commons Attribution License, which permits unrestricted use, distribution, and reproduction in any medium, provided the original work is properly cited.

\begin{abstract}
Transcatheter arterial chemoembolization (TACE) is one of the effective treatment methods for hepatocellular carcinoma (HCC) in middle and late phases. However, TACE-induced hypoxia may promote the angiogenesis and section of some cytokines, such as IL-8, and, thereby, lead to tumor metastasis. Therefore, we investigated the effect of Jiedu Recipe (JR), which has been demonstrated as an effective Traditional Chinese Medicine (TCM) recipe on HCC, on TACE-induced cytokines upregulation and hypoxia-induced angiogenesis. A total of 88 hepatocellular carcinoma (HCC) patients treated with TACE were enrolled and divided into a JR group or control group. TACE induced significant increases of neutrophil lymphocyte ratio (NLR), platelet lymphocyte ratio (PLR), IL-1 $\beta$, IL-2R, IL-6, and IL-8. JR treatment significantly inhibited the elevation of IL- 8 compared with control. In vitro, JR significantly inhibited the hypoxia-induced overexpression of IL-8, HIF-1 $\alpha$, and VEGF mRNA in Huh 7 cells. ELISA assay demonstrated the effect of JR on IL-8 expression. Both hypoxia and IL-8 may promote angiogenesis which was suppressed by JR. Western blot showed that IL- 8 upregulated the expression of phosphorylation of AKT, ERK, NF- $\kappa$ B, and VEGFR, which were inhibited by JR. On the other hand, effects of IL-8 on the increase of p-AKT and p-ERK were also blocked by LY294002 and U0126, respectively. In conclusion, our results indicated that JR may inhibit hypoxia-induced angiogenesis through suppressing IL-8/HIF-1 $\alpha /$ PI3K and MAPK/ERK pathways after TACE in HCC patients.
\end{abstract}

\section{Introduction}

Hepatocellular carcinoma (HCC) accounts for $>80 \%$ of liver cancer, which occurs worldwide with 782,500 new cases annually $[1,2]$. HCC surveillance and early detection may increase the opportunity of curative treatments such as surgical resection, local ablation, or liver transplantation [3]. However, most HCC patients are diagnosed middle and late phases due to the aggressive growth and late symptom presentation. For middle-phase HCC patients, transcatheter arterial chemoembolization (TACE) is an effective treatment option. In TACE, embolic material, usually the lipiodol, is injected to block the tumor-feeding artery through the hepatic artery. However, clinical practice and previous studies showed that complete embolization of tumor is usually impossible $[4,5]$. In the tumor microenvironment, incomplete embolization results in relative hypoxia and, thereby, elevates the expression of hypoxia-inducible factor$1 \alpha(\mathrm{HIF}-1 \alpha)[6,7]$.

In HCC, the role of HIF- $1 \alpha$ has been widely investigated. Overexpression of HIF- $1 \alpha$ induces the upregulation of angiogenic growth factors such as VEGF and promotes formation of tube by vascular endothelial cells and vasculogenic mimicry by tumor cells [8-10]. In addition, HIF- $1 \alpha$ 
also involves in the induction of some inflammatory cytokines, such as IL- $8[11,12]$, which may also promote the expression of VEGF independently [13]. Although multikinase inhibitor sorafenib may target the VEGF receptors (VEGFRs), platelet-derived growth factor receptor (PDGFR), etc., which are key factors of angiogenesis, its combination with TACE did not improve time-to-tumor progression in a clinically meaningful manner when compared with TACE alone [14]. Another multikinase inhibitor lenvatinib showed the same result [15]. Therefore, neoangiogenic mechanisms after TACE is complex, and novel treatments are urgently needed.

Traditional Chinese medicine, mainly used in China for thousands of years, has been demonstrated by many studies that it may retard HCC progression, improve quality of life in HCC patients, and prevent HCC occurrence either alone or in combination with other conventional therapies, including TACE [16-18]. Jiedu Recipe (JR), which contains pseudobulbus cremastrae seu pleiones, valvate actinidia root, salvia chinensis, and endothelium corneum gigeriae galli, may prolong the survival of patients with advanced HCC [19]. Our retrospective study also indicated that TACE combined with JR may improve the prognosis and prolong survival of patients with unresectable HCC when compared to the patients treated with TACE alone [20].

However, the mechanisms beyond JR have not been fully investigated. Therefore, the aim of the present study was to explore the effect of JR on HCC, on TACE-induced cytokines upregulation, and hypoxia-induced angiogenesis.

\section{Materials and Methods}

2.1. Study Design. We conducted a prospective cohort study of 88 patients diagnosed with liver cancer and treated with TACE at Changhai Hospital, Second Military Medical University (Shanghai, China), from February 2018 through August 2018. Eligible participants for this study were over 18 years of age; diagnosed with liver cancer and treated with TACE; Child-Pugh grade A or B; and did not receive any herbal or systemic treatment for 2 weeks prior to the start of the trial. Patients who received treatment that may affect the effect of the trial; who participated in other clinical trials; and who had other serious diseases that may affect treatments in the trial were excluded.

Enrolled patients were assigned to the control group or JR group randomly after providing written informed consent. All patients received TACE treatment in Changhai Hospital, and supportive care such as liver-protecting treatment and antiemetics was permitted in the both groups. Besides, patients in the JR group may take JR at a dose of $4.5 \mathrm{~g}$ orally twice daily for 2 months (JR was prepared with herbs of pseudobulbus cremastrae seu pleiones, valvate actinidia root, salvia chinensis, and endothelium corneum gigeriae galli in a ratio of $2.5: 2.5: 1: 1$ by Jiangyin Tianjiang Pharmaceutical Company, Jiangsu, China).

Concentration of serum IL- $1 \beta$, IL- 6 , IL- 8, TNF- $\alpha$, platelet lymphocyte ratio (PLR), and neutrophil lymphocyte ratio (NLR) were detected before and 3 days and 2 months after TACE.
The protocols of the present trial were approved by the First Affliated Changhai Hospital of Second Military Medical University Ethics Committee (Shanghai, China) (Approval No: CHEC2018-074).

2.2. Preparation of the JR. The JR lyophilized powder was produced by Shanghai Winherb Medical Technology Co., Ltd. (Shanghai, China) through extracting herbs of pseudobulbus cremastrae seu pleiones, valvate actinidia root, salvia chinensis, and endothelium corneum gigeriae galli in a ratio of $2.5: 2.5: 1: 1(\mathrm{w} / \mathrm{w})$. In detail, the abovementioned dried and pulverized herbs were mixed together and were extracted with $85 \%$ ethanol by heat under reflux. This step was repeated twice, and the extracts were mixed. Then, the mixture was filtrated and further concentrated under vacuum at $50^{\circ} \mathrm{C}$, productions from which were freeze-dried (with cold trap temperature $-56^{\circ} \mathrm{C}$ ) to obtained the lyophilized powder and stored at $4^{\circ} \mathrm{C}$ before used. For quality control, high-performance liquid chromatography assay was performed to determine the fingerprinting of JR in the School of Pharmacy, Second Military Medical University. In the in vitro assays, JR was dissolved in the cell culture medium and filtered twice with a $0.22 \mu \mathrm{m}$ filter.

2.3. Cell Lines. The human HCC cell line Huh 7 and human immortalized endothelial cells, EA.hy 926, were obtained from the Cell Bank of the Chinese Academy of Sciences Committee Type Culture Collection (Shanghai, China). The cells were cultured in high glucose Dulbecco's modified Eagle's medium (DMEM) (Thermo, Shanghai, China) supplemented with $10 \%$ fetal bovine serum (FBS, Gibco Life Technologies, Carlsbad, CA, USA), $100 \mathrm{U} / \mathrm{mL}$ penicillin, and $0.1 \mathrm{mg} / \mathrm{mL}$ streptomycin (Hyclone, Life Sciences, USA). The cells were cultured in a humidified atmosphere of $95 \%$ normal air and $5 \% \mathrm{CO}_{2}$ at $37^{\circ} \mathrm{C}$. Cells were passaged after cell density reaching $80 \%$ confluence and used in the experiment as in their logarithmic growth phase.

2.4. Cell Viability MTT Assay. To examine the effect of JR on cell growth, EA.hy 926 cells were seeded into 96-well plates with $5 \times 10^{3} /$ well and cultured overnight. Then, cells were treated with varying concentrations of the JR $(0.2,0.4,0.8$, $1.6 \mathrm{mg} / \mathrm{mL}$ ) for 24 or $48 \mathrm{~h}$, and $20 \mu \mathrm{L}$ MTT solution $(5 \mathrm{mg} /$ $\mathrm{mL}$ ) was added to each well and incubated at $37^{\circ} \mathrm{C}$ for an additional $4 \mathrm{~h}$. After carefully removing the culture medium, $150 \mu \mathrm{L}$ dimethyl sulfoxide was added to each well to dissolve the crystals. A multiskan spectrum microplate reader (Thermo Fisher Scientific, Waltham, MA, USA) was used to measure absorbance of the converted dye in living cells at a wavelength of $570 \mathrm{~nm}$.

2.5. Capillary-Like Tube Formation Assay. The capillary-like tube formation assay was used to evaluate angiogenesis as described in the previous studies with modification [21]. Briefly, Matrigel (BD Biosciences, USA) was thawed at $4^{\circ} \mathrm{C}$ overnight before it was administered into the cold 96-well plate with $50 \mu \mathrm{L}$ gel/well and incubated at $37^{\circ} \mathrm{C}$ for one hour. 
Then, EA.hy 926 cells suspended in serum-free DMEM with a concentration of $5 \times 10^{5} / \mathrm{mL}$ were plated to the plate with $100 \mu \mathrm{L} /$ well. Cells were treated with cobalt chloride $\left(\mathrm{CoCl}_{2}\right)$, which was used to induce hypoxia-like condition, or human IL-8 protein (R\&D Systems, USA) or JR. After $16 \mathrm{~h}$ incubation at $37^{\circ} \mathrm{C}$, tube formation ability of the cells was visualized under the inverted phase contrast microscope (Leica, Germany) and evaluated by counting the number of junctions, total segments length, and mean mesh size with the open source software Image J (version 1.51).

2.6. Real-Time Reverse Transcription-Polymerase Chain Reaction Assay. Total RNA was isolated from Huh 7 cells by using Trizol reagent (Invitrogen, USA) as described previously [22]. The first-strand cDNA was synthesized using the PrimeScript RT reagent Kit (Takara, Japan) according to the manufacturer's procedures. qPCR was performed in a CFX96 Real-Time PCR system (Bio-Rad, CA, USA) using a commercial SYBR Green PCR Master Mix (TOYOBO, Osaka, Japan). The relative gene expression was measured and normalized to $\beta$-actin by the $2^{-\Delta \Delta \mathrm{Ct}}$ method. The primers used in this experiment were as follows: HIF- $1 \alpha$, forward: $5^{\prime}$-TTC CCG ACT AGG CCC ATT C-3', reverse: 5'-CAG GTA TTC AAG GTC CCA TTT CA-3'; VEGF, forward: 5'-GCC TCG GGC TTG TCA CAT TTT-3', reverse: $5^{\prime}$-CCC TGA TGA GAT CGA GTA CAT CT-3'; IL-8, forward: $5^{\prime}$-TCT TGG CAG CCT TCC TGA TT-3', reverse: $5^{\prime}$-TGG TCC ACT CTC AAT CAC TCT CAG T-3'; And $\beta$-actin, forward: $5^{\prime}$-AGC GGG AAA TCG TGC GTG-3', reverse: $5^{\prime}$-CAG GGT ACA TGG TGG TGC C-3'.

\subsection{Enzyme-Linked Immunosorbent Assay (ELISA).} Protein levels of IL- 8 in cell culture supernatants were measured with ELISA assay. Huh 7 cells were treated with $\mathrm{CoCl}_{2}$ with or without $0.2,0.4$, and $0.8 \mathrm{mg} / \mathrm{mL}$ JR for $24 \mathrm{~h}$. Cell culture media were then collected and centrifuged at $1500 \times \mathrm{g}$ for $20 \mathrm{~min}$ to obtain the supernatants. ELISA assay was carried out with Human IL-8 ELISA kits (R\&D Systems, USA) to determine the IL-8 levels in the obtained supernatants according to the manufacturer's instructions.

2.8. Western Blot Assay. Total protein was extracted from treated EA.hy 926 or Huh 7 cells with cell lysis buffer (Cell Signaling Technology, USA) containing protease and phosphatase inhibitors, and protein quantification was carried out with a BCA protein assay kit (Thermo, USA). Equivalent protein content was then separated by electrophoresis and transferred to the PVDF membranes. The membranes were first blocked for $1 \mathrm{~h}$ with $5 \%$ skim milk and then incubated with primary antibodies overnight. After incubated with horseradish peroxidase-conjugated secondary antibodies for $2 \mathrm{~h}$ at room temperature, target protein blots were detected by enhanced chemiluminescence reagents (Thermo, USA).

2.9. Statistical Analysis. SPSS 19.0 software was used for all statistical analyses, and data were presented as mean \pm standard deviation. For the clinical data, Student's $t$ test or chi-square test was used to compare the difference between the groups and Fisher's exact test was used for analysis of categorical variables. For the in vitro assay, oneway ANOVA followed by Tukey's test was used for the analysis. All results were considered statistically significant when $P<0.05$.

\section{Results}

3.1. Patient Characteristics. A total of 88 patients meeting the study criteria were enrolled with 47 in the control group and 41 in the JR group. The baseline data for the patients in the 2 groups were comparable in terms of age, gender, Child-Pugh score, performance status, and BCLC stage (Table 1).

3.2. JR Inhibited IL-8 Elevation after TACE. The changes of inflammatory cytokines before and after TACE were examined to determine the target of JR. As shown in Figure 1, NLR, PLR, and all the detected cytokines, including IL- $1 \beta$, IL-2R, IL-6, and IL-8, were significantly upregulated on the 3rd day in the control group after TACE compared to those before TACE $(P<0.01)$. On the 3rd day after TACE, the levels of PLR and IL- 8 were lower in the JR group than the control group, indicating JR administration may suppress TACE-induced upregulation of PLR and IL-8 (Figure 1).

3.3. Effect of JR on Endothelial Cells Proliferation. To explore whether JR may inhibit angiogenesis in vitro, MTT assay was firstly conducted to evaluate the effects of JR $(0,0.2,0.4,0.8$, and $1.6 \mathrm{mg} / \mathrm{ml}$ ) on the proliferation of human immortalized endothelial cells EA.hy 926. Data presented in Figure 2(a) showed that, after treatment for $24 \mathrm{~h}$, JR at the concentration of $1.6 \mathrm{mg} / \mathrm{mL}$ significantly inhibited the proliferation of EA.hy 926 cells compared with control $(P<0.05)$ (Figure 2(a)). When treated for $48 \mathrm{~h}$, JR at the concentration of $0.4-1.6 \mathrm{mg} / \mathrm{mL}$ significantly suppressed the endothelial cells proliferation (Figure 2(b)). However, the maximum inhibitory rate was only $18.29 \%$ for JR.

\subsection{Effect of JR on Capillary-Like Tube Formation under} Hypoxic Condition. Effect of JR on the angiogenesis was evaluated by capillary-like tube formation assay in vitro. Hypoxia induced by $\mathrm{CoCl}_{2}$ significantly stimulated the formation of capillary-like tube, which was significantly attenuated by JR treatment (Figure 3(a)). In detail, we used the open source software Image J to mark out the tubule branches (Figure 3(a)) and calculated the number of junctions, total segments length, and mean mesh size to assess the tube formation ability quantitatively (Figures 3(b)-3(d)). $\mathrm{CoCl}_{2}$ treatment significantly increased the number of junctions and total segments length compared with the control group. Also, JR significantly decreased the number of junctions and total segments length at both 0.2 and $0.4 \mathrm{mg} /$ $\mathrm{ml}$ (Figures 3(b) and 3(c)). 
TABLE 1: Baseline of enrolled patients.

\begin{tabular}{|c|c|c|c|c|c|c|c|c|}
\hline \multirow{2}{*}{ Variable } & \multicolumn{2}{|c|}{ JR } & \multicolumn{2}{|c|}{ Control } & \multicolumn{2}{|c|}{ Total } & \multirow{2}{*}{$Z$ value } & \multirow{2}{*}{$P$ value } \\
\hline & $N$ & Percent & $N$ & Percent & $N$ & Percent & & \\
\hline Age (years) & & & & & & & 1.40 & 0.16 \\
\hline$<55$ & 14 & 34.1 & 23 & 48.9 & 37 & 42 & & \\
\hline$\geq 55$ & 27 & 65.9 & 24 & 51.1 & 51 & 58 & & \\
\hline Gender & & & & & & & 0.99 & 0.33 \\
\hline Male & 37 & 90.2 & 39 & 83 & 76 & 86.4 & & \\
\hline Female & 4 & 9.8 & 8 & 17 & 12 & 13.6 & & \\
\hline Child-Pugh score & & & & & & & 0.85 & 0.40 \\
\hline A & 31 & 75.6 & 39 & 83 & 70 & 79.5 & & \\
\hline $\mathrm{B}$ & 10 & 24.4 & 8 & 17 & 18 & 20.5 & & \\
\hline Performance status & & & & & & & 0.47 & 0.64 \\
\hline Score 0 & 40 & 97.6 & 45 & 95.7 & 85 & 96.6 & & \\
\hline Score 1 & 1 & 2.4 & 2 & 4.3 & 3 & 3.4 & & \\
\hline BCLC stages & & & & & & & 1.51 & 0.13 \\
\hline Stage A & 6 & 14.6 & 5 & 10.6 & 11 & 12.5 & & \\
\hline Stage B & 25 & 61 & 23 & 48.9 & 48 & 54.5 & & \\
\hline Stage C & 10 & 24.4 & 19 & 40.4 & 29 & 33 & & \\
\hline
\end{tabular}
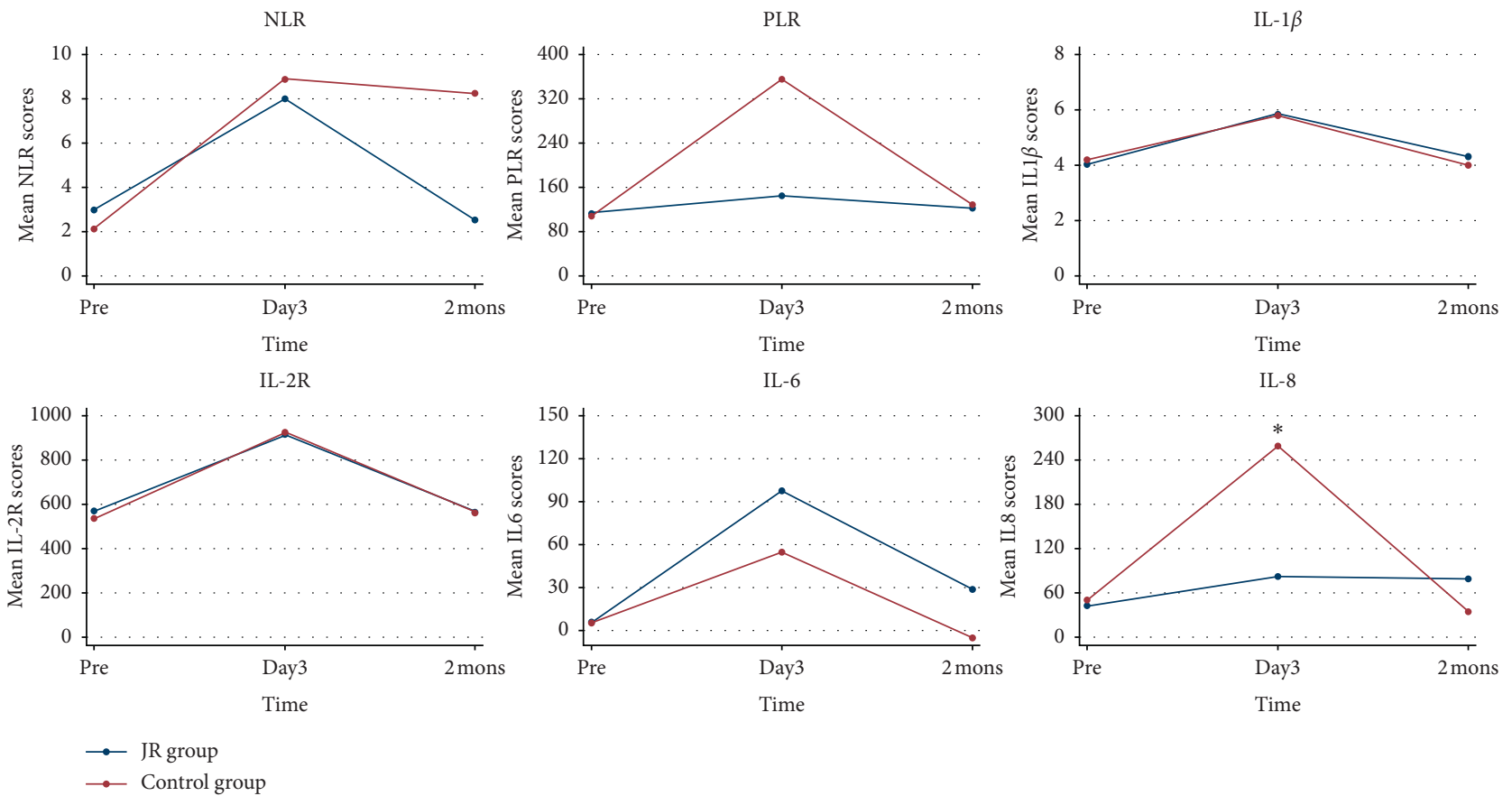

FIGURE 1: Changes of inflammatory cytokines before and after TACE. NLR: neutrophil lymphocyte ratio; PLR: platelet lymphocyte ratio; IL: interleukin; IL-2R: interleukin-2 receptor. ${ }^{*} P<0.05$, compared with the control group.

3.5. Effect of JR on the Expression of VEGF and IL-8 under Hypoxic Condition. Since the expression of VEGF and IL- 8 is upregulated in the tumor hypoxic microenviroment, we then detected the transcriptional levels of VEGF and IL-8 in $\mathrm{HCC}$ cells under hypoxic condition. $\mathrm{CoCl}_{2}$ treatment significantly upregulated the expression of VEGF and IL-8 mRNA. Treatment with different concentrations of JR significantly inhibited the levels of VEGF and IL- 8 mRNA in a dose-dependent manner (Figure 4).

We further confirmed the changes of IL- 8 by ELISA. Under hypoxic condition, the concentration IL-8 significantly increased in the supernatant of Huh 7 cells. It was also antagonized by JR in a dose-dependent way $(P<0.05)$ (Figure 5).

3.6. JR Inhibited IL-8-Induced Angiogenesis through PI3K/ $A K T$ and MAPK/ERK Signals. It has been reported that IL-8 is able to induce the expression of VEGF independently [13] and the coexpression of IL- 8 and HIF- $1 \alpha$ is associated with metastasis and poor prognosis in HCC [23]. Therefore, we then observed whether IL-8 could promote the tube 
$24 \mathrm{~h}$

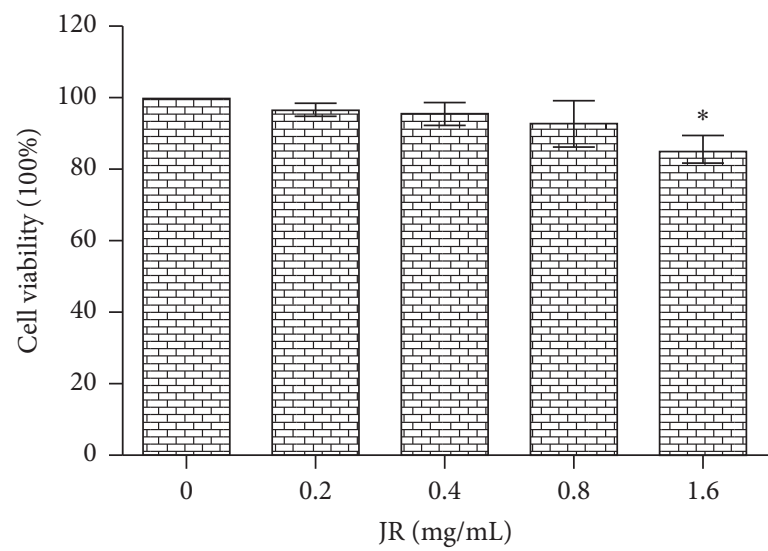

(a)

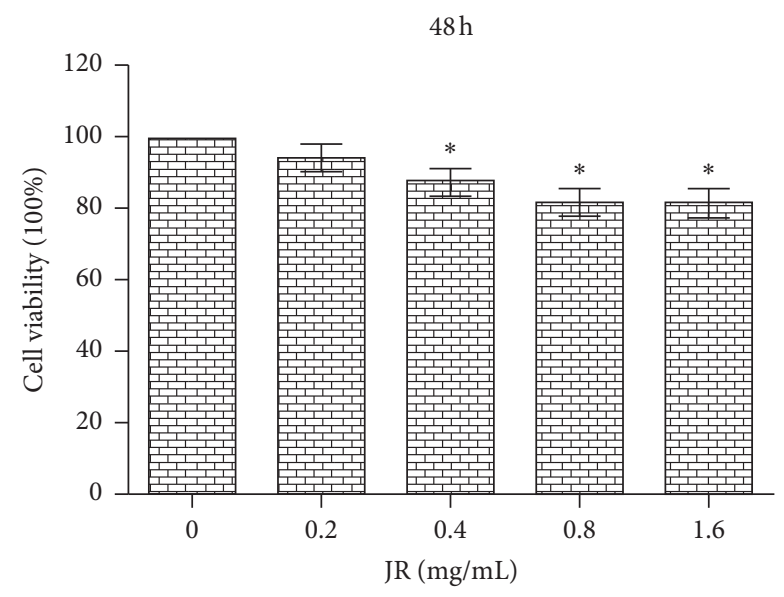

(b)

FIGURE 2: Effect of JR on EA.hy 926 cells' proliferation. MTT assay was used to determine the cell proliferation. EA.hy 926 cells were treated with $0.2,0.4,0.8$, and $1.6 \mathrm{mg} / \mathrm{mL}$ JR for $24 \mathrm{~h}$ (a) and $48 \mathrm{~h}$ (b). Data are expressed as means \pm S.D. ${ }^{*} P<0.01$ vs. control group.
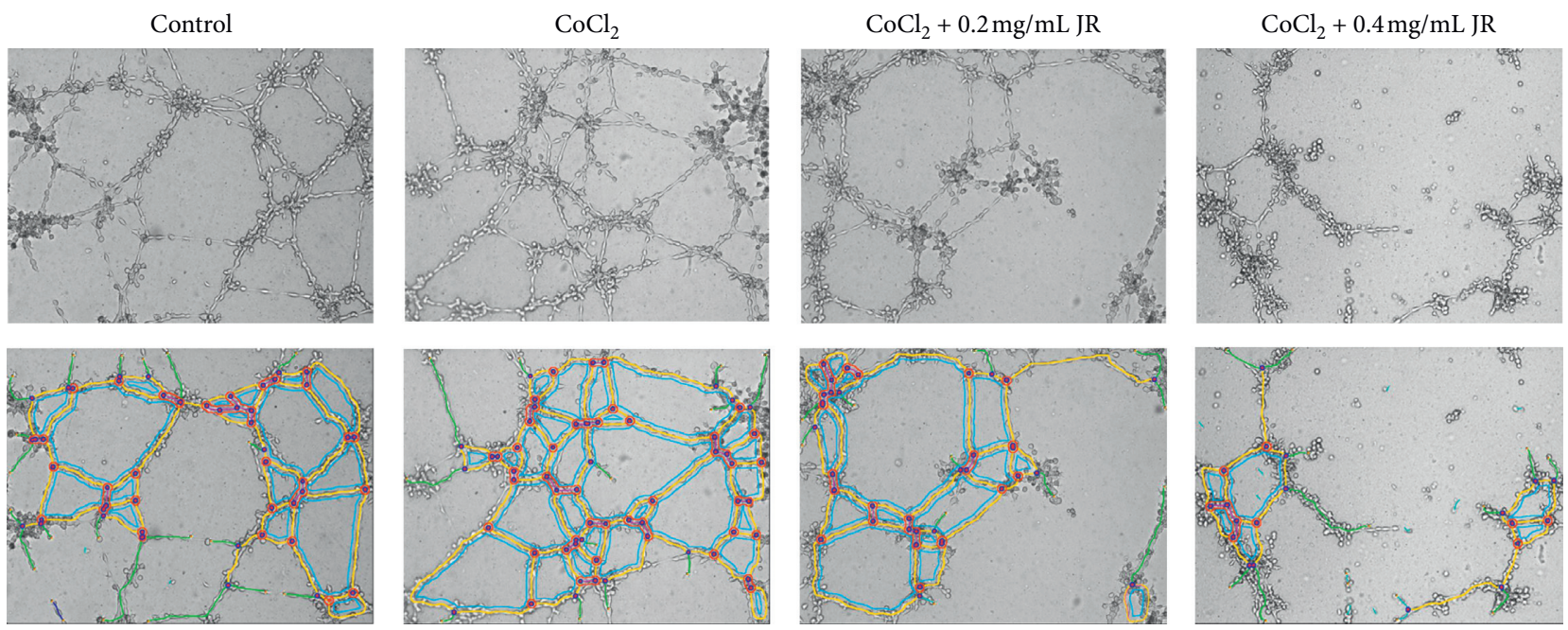

(a)

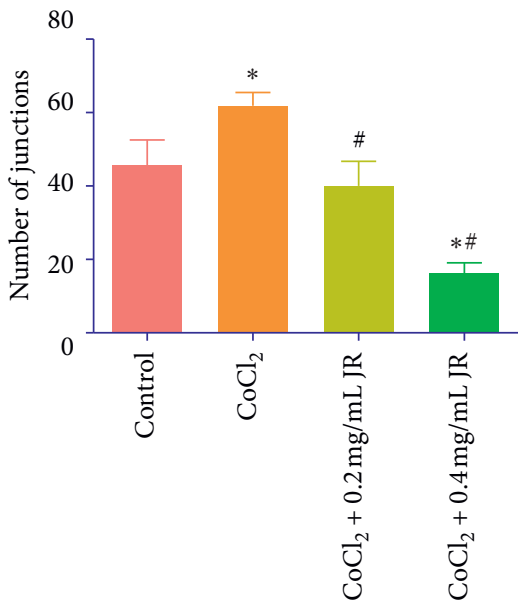

(b)

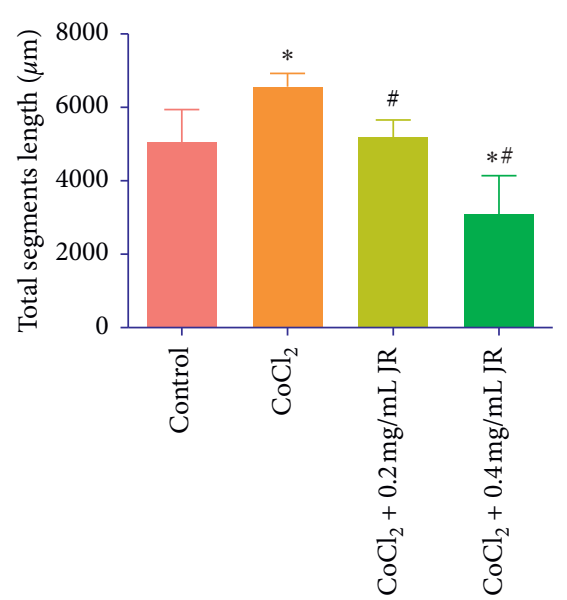

(c)

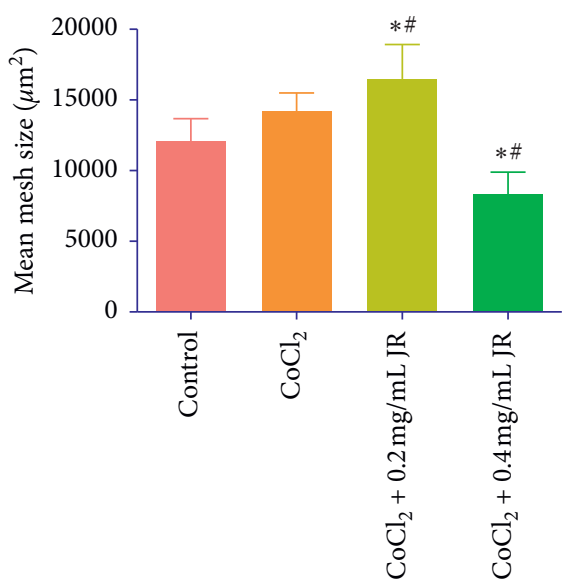

(d)

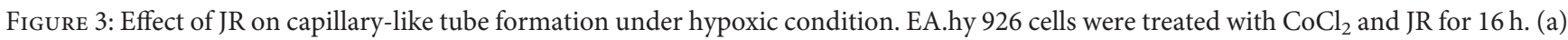
Inverted phase-contrast microscopy images of capillary-like tube formation and tubule branches marked out by Image J software (magnification of 100x). ((b)-(d)) Number of junctions, total segments length, and mean mesh size of the tube, respectively. Data are expressed as means \pm S.D. ${ }^{*} P<0.05$ vs. control group and ${ }^{\#} P<0.05$ vs. $\mathrm{CoCl}_{2}$ group. 


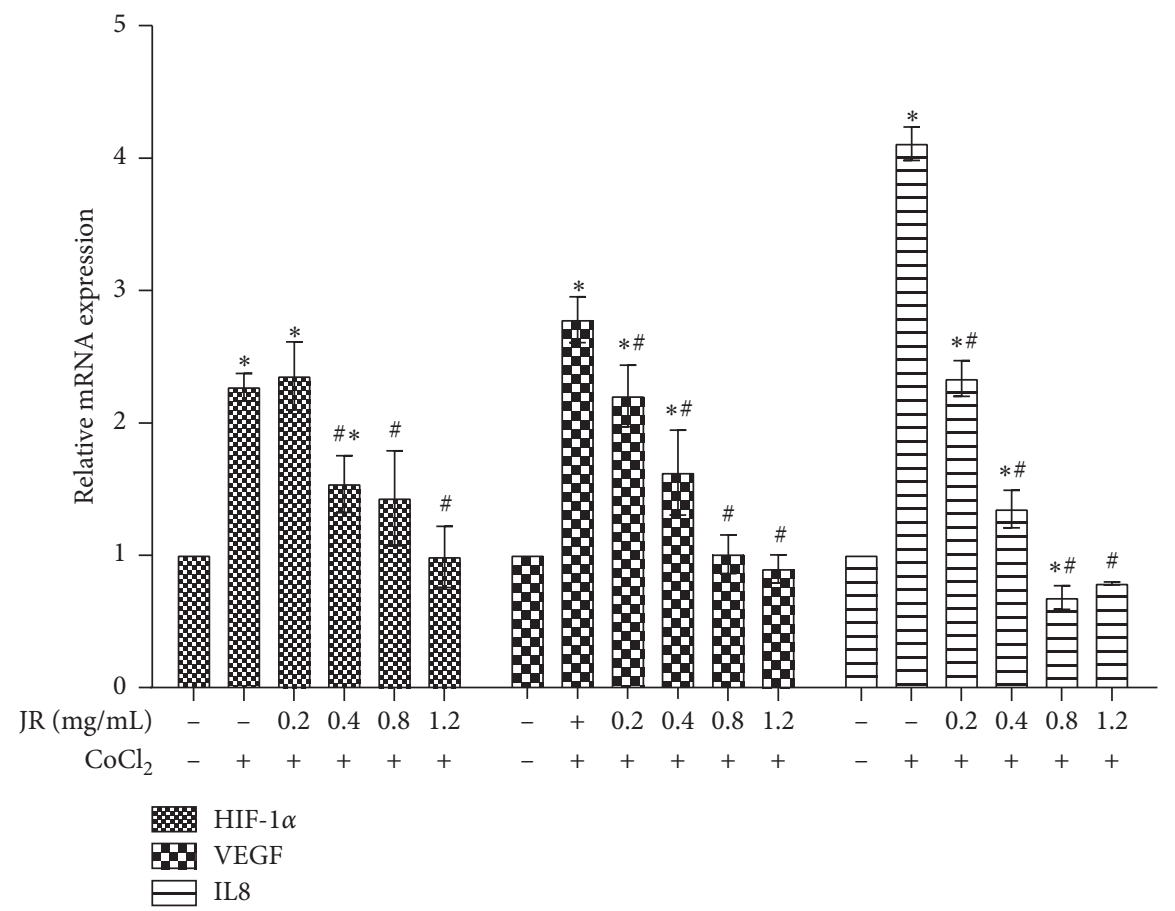

Figure 4: Effect of JR on the mRNA expression of VEGF and IL-8 under hypoxic condition. Huh 7 cells were treated with CoCl ${ }_{2}$ and JR for $24 \mathrm{~h}$, and mRNA expression of HIF-1 $\alpha$, VEGF, and IL- 8 was detected. Data are expressed as means \pm S.D. and ${ }^{*} P<0.05$ vs. control group and ${ }^{\#} P<0.05$ vs. $\mathrm{CoCl}_{2}$ group.

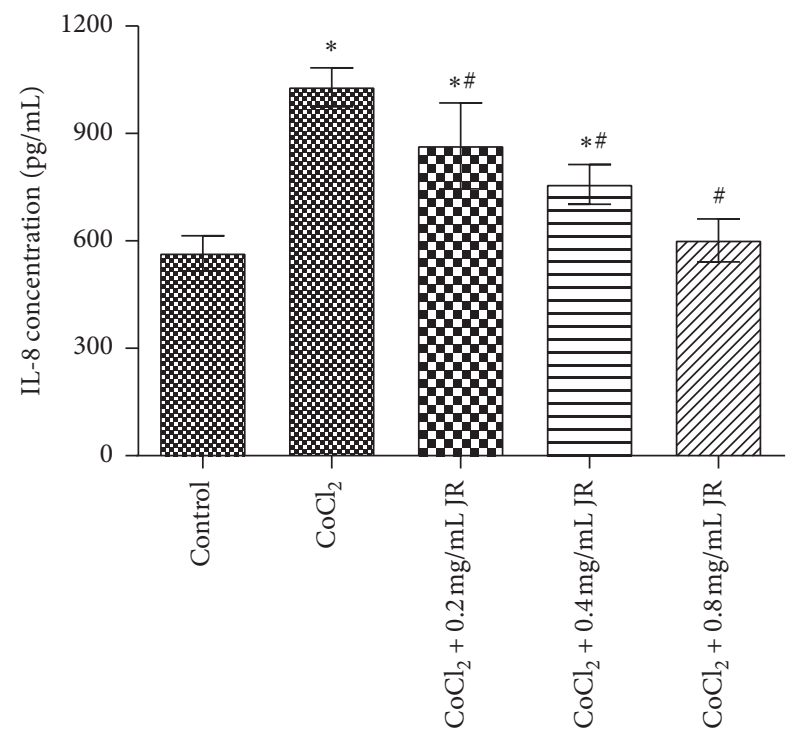

FIGURE 5: Effect of JR on the IL-8 protein secretion under hypoxic condition. Huh 7 cells were treated with $\mathrm{CoCl}_{2}$ and JR for $24 \mathrm{~h}$, and ELISA assay was used to determine the IL- 8 proteins in the culture media. Data are expressed as means \pm S.D. and ${ }^{*} P<0.05$ vs. control group and ${ }^{\#} P<0.05$ vs. $\mathrm{CoCl}_{2}$ group.

formation directly. IL-8 stimulation significantly promoted the tube formation of EA.hy 926 cells, which was suppressed by both 0.2 and $0.4 \mathrm{mg} / \mathrm{mL}$ of JR (Figure 6).

Western blot showed that IL-8 upregulated the phosphorylation of AKT, ERK, and NF- $\kappa \mathrm{B}$, which was inhibited by JR. JR may also inhibit the IL-8-induced upregulated
VEGFR. On the other hand, effects of IL- 8 on the p-AKT and p-ERK were also demonstrated by the inhibitor LY294002 and U0126, respectively (Figure 7).

\section{Discussion}

TACE is currently recognized as the preferred therapy for the unresectable liver cancer and provides the modest survival benefit [24]. The hypoxic microenvironment plays an important role in the tumor cell survival and metastasis and rapid formation of blood vessels after TACE. In addition, hypoxia-induced inflammatory cytokines, such as IL8, stimulate angiogenesis, which may be an independent risk factor on HCC progression, metastasis, and recurrence [25-27]. In this study, we showed that JR may inhibit hypoxia-induced angiogenesis through suppressing IL-8/ HIF- $1 \alpha / \mathrm{PI} 3 \mathrm{~K}$ and MAPK/ERK pathways after TACE in HCC patients.

TACE delivers lipiodol and chemotherapeutic drugs to obstruct tumor-feeding arteries and, thereby, induces a hypoxic microenvironment [28]. In the present study, $\mathrm{CoCl}_{2}$ was used to induce hypoxia-like condition and stimulated the formation of capillary-like tube, which was attenuated by JR treatment without inhibiting the proliferation of EA.hy 926 cells. HIF- $1 \alpha$ is a heterodimer protein comprising an oxygen-sensitive $\alpha$ subunit and plays a critical role in regulating cellular responses to hypoxia and promotes angiogenesis by activating the expression of downstream-related genes such as VEGF $[29,30]$. Our results also indicate that $\mathrm{CoCl}_{2}$ may upregulate the expression of HIF- $1 \alpha$ and VEGF. By treating with different concentrations of JR, the levels of 

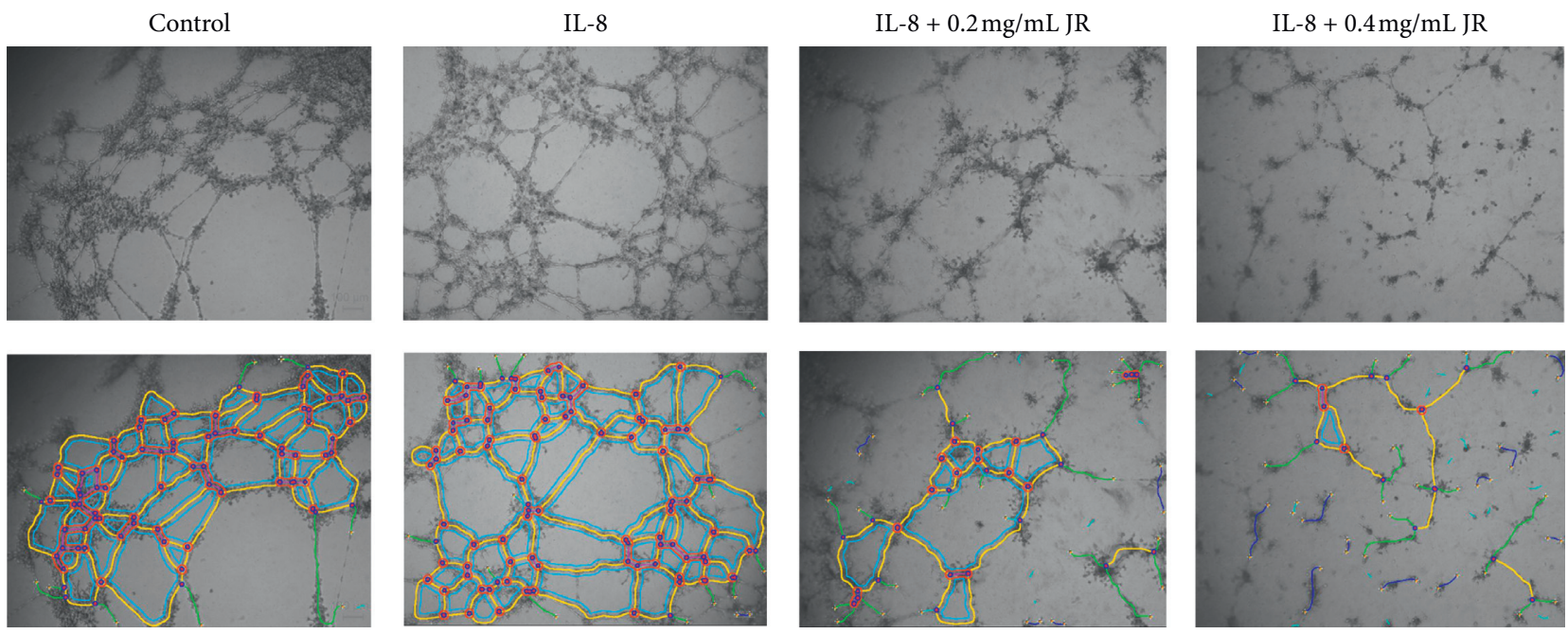

(a)

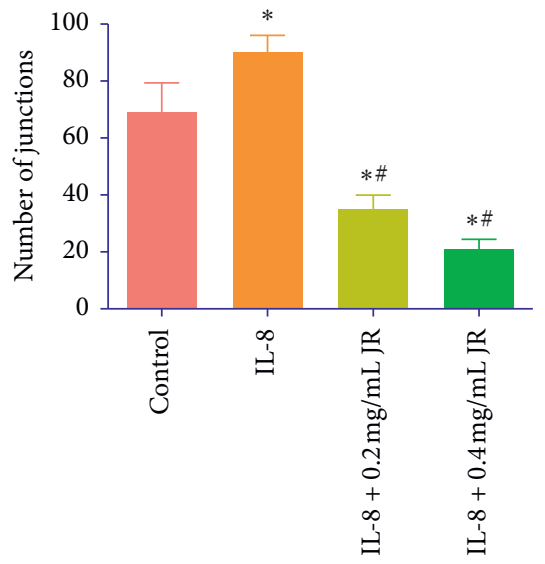

(b)

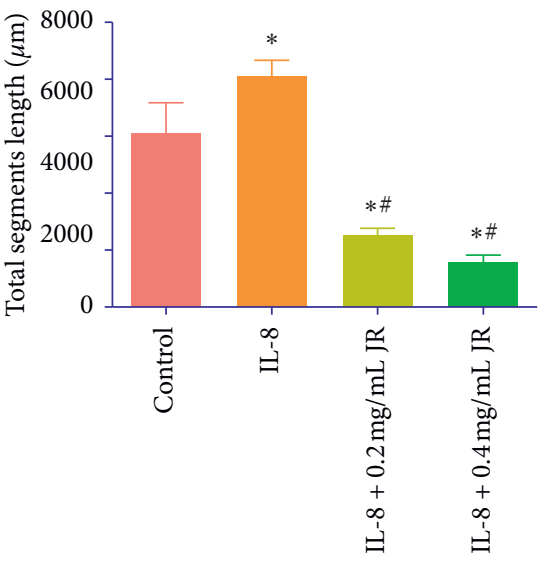

(c)

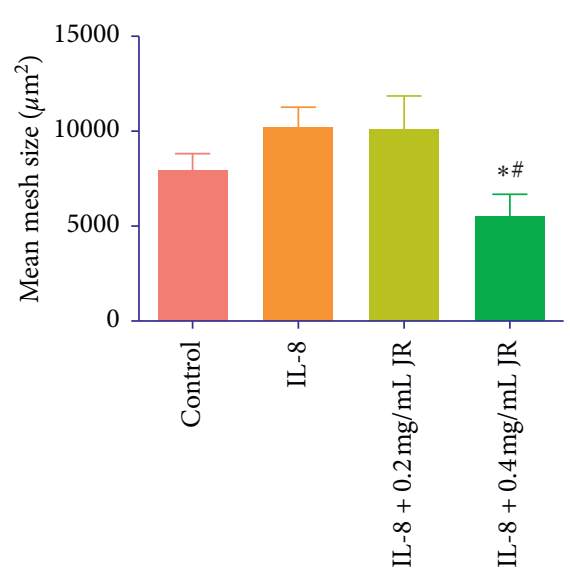

(d)

FIgURE 6: Effect of JR on IL-8-induced capillary-like tube formation. EA.hy 926 cells were treated with human IL-8 protein and JR for 16 h. (a) Inverted phase-contrast microscopy images of capillary-like tube formation and tubule branches marked out by Image J software (magnification of 100x). ((b)-(d)) Number of junctions, total segments length, and mean mesh size of the tube, respectively. Data are expressed as means \pm S.D. ${ }^{*} P<0.05$ vs. control group and ${ }^{\#} P<0.05$ vs. IL-8 group.

HIF- $1 \alpha$ and VEGF were significantly inhibited in a dosedependent way upon the hypoxic condition.

The response rates to TACE are heterogeneous since many factors may affect the prognosis of the disease [31]. Kim et al. [32] showed that IL-6 and IL-22 increase early after TACE. In our study, we detected the levels of several cytokines, including IL-1 $\beta$, IL-2R, IL-6, and IL-8, which were all increased after TACE. PLR and NLR, which may represent convenient, quick, and noninvasive biological markers for the prognostic prediction in HCC [33-35], were also studied in this study. Interestingly, JR may downregulate the PLR in the early stage but in the late stage for NLR after TACE. It seems that JR performs a better role in PLR. Several reports have indicated that high IL- 8 level correlates with reduced overall survival and may be served as a prognostic index independent of the target lesions' size or the patients' liver function or age [36, 37]. In this study, we found the JR suppressed the elevated IL-8 levels after TACE.
These results indicate that JR may improve the prognosis of HCC patients through inhibiting hypoxia-induced angiogenesis in the HCC microenvironment.

IL-8 may predict poor prognosis in HCC when coexpressed with HIF- $1 \alpha[23,38]$. In another study, the relationship of HIF- $1 \alpha$ and IL- 8 was evidenced by the downregulation of IL- 8 in response to silencing of HIF- $1 \alpha$ in HCC cell lines under hypoxic condition [39]. IL-8 knockdown inhibits angiogenesis and tumor growth in HCC independent of HIF- $1 \alpha$ [40]. In our study, JR inhibited IL-8 upregulation under hypoxic condition and IL-8-induced capillary-like tube formation. Furthermore, phosphorylation of AKT, ERK, and NF- $\kappa \mathrm{B}$ was closely related to the tumor metastasis and angiogenesis [41], which was demonstrated in our study that IL-8 may stimulate the upregulation of p-AKT, p-ERK, and p-NF- $\kappa$ B, which were inhibited by both JR. All our abovementioned results suggest that JR may inhibit the angiogenesis upon hypoxic condition through 


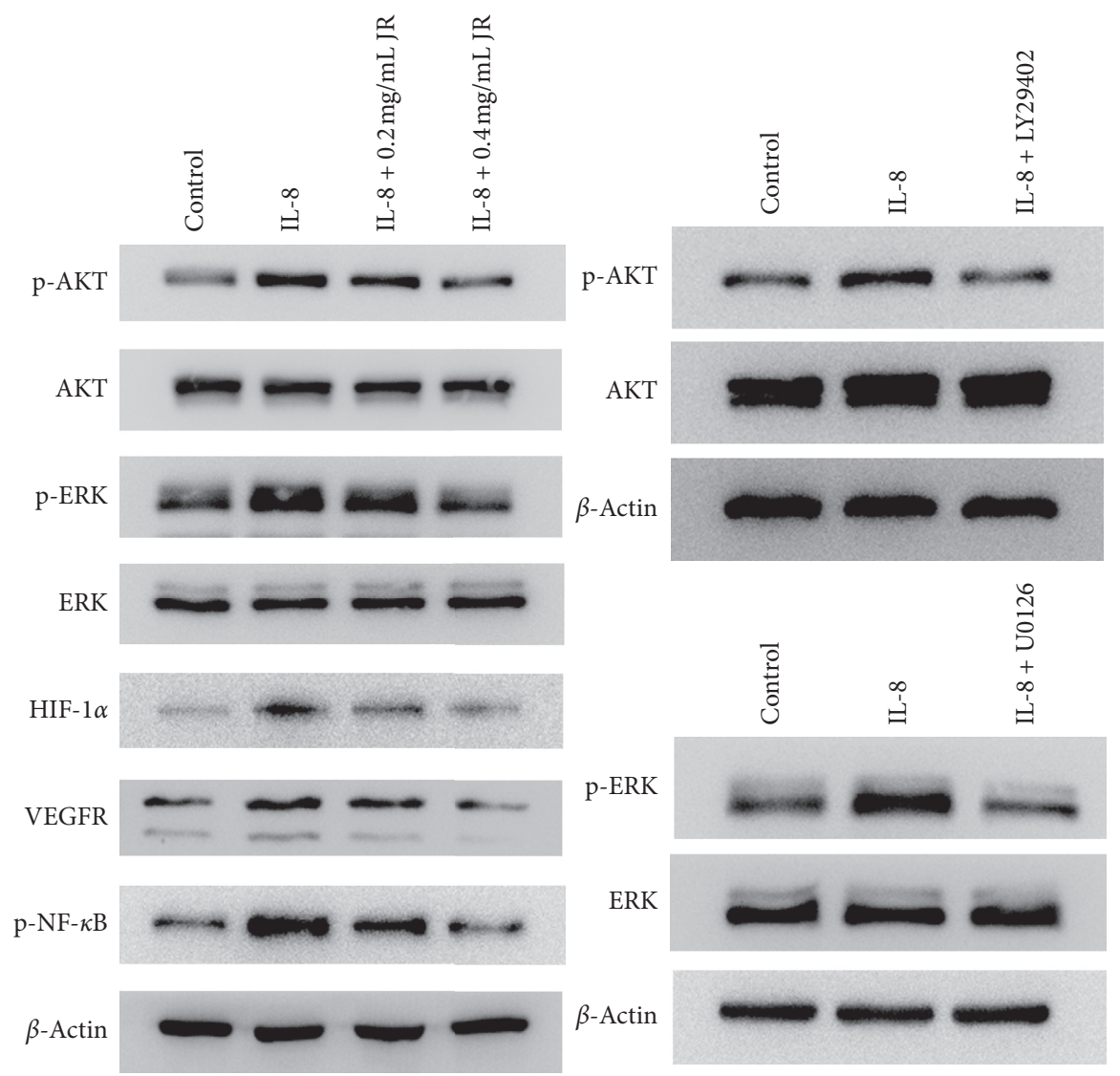

FIGURE 7: Effect of JR on p-AKT and p-ERK expression induced by IL- 8 . Western blot assay was used to detect the p-AKT, p-ERK p-NF- $\kappa$ B, and VEGFR, the expression of p-AKT after treated with human IL-8 protein and PI3K inhibitor LY294002, and the expression of p-ERK after treated with human IL-8 protein and ERK inhibitor U0126. $\beta$-Actin served as the loading control.

IL-8/HIF- $1 \alpha /$ PI3K and MAPK/ERK pathways. Further study is needed to provide a more exact mechanism of the effects of JR and promote its clinical application.

\section{Conclusions}

In conclusion, JR can reduce the expression of many inflammatory cytokines in HCC patients after TACE. JR inhibits hypoxia-induced angiogenesis through suppressing IL-8/HIF- $1 \alpha /$ PI3K and MAPK/ERK pathways after TACE in HCC patients. Also, further study is needed to provide more exact mechanism of the effects of JR and confirm its clinical efficacy.

\section{Data Availability}

The data used to support the findings of this study are available from the corresponding author upon request.

\section{Conflicts of Interest}

The authors declare that there are no conflicts of interest regarding the publication of this paper.

\section{Authors' Contributions}

Wanfu Lin, Huan Wang, and Maofeng Zhong contributed equally to this work and should be considered as co-first authors.

\section{Acknowledgments}

This work was supported by the grants from the scientific research project of Science and Technology Commission of Shanghai Municipality (grant no. 18401901400) and the National Natural Science Foundation of China (grant nos. 81430101, 81673655, and 81774077).

\section{References}

[1] Global Burden of Disease Cancer, C. Fitzmaurice, C. Allen et al., "Global, regional, and national cancer incidence, mortality, years of life lost, years lived with disability, and disability-adjusted life-years for 32 cancer groups, 1990 to 2015: a systematic analysis for the global burden of disease study," JAMA Oncology, vol. 3, no. 4, pp. 524-548, 2017.

[2] R. L. Siegel, K. D. Miller, and A. Jemal, "Cancer statistics, 2017," CA: A Cancer Journal for Clinicians, vol. 67, no. 1, pp. 7-30, 2017. 
[3] J. D. Yang, P. Hainaut, G. J. Gores, A. Amadou, A. Plymoth, and L. R. Roberts, "A global view of hepatocellular carcinoma: trends, risk, prevention and management," Nature Reviews Gastroenterology \& Hepatology, vol. 16, no. 10, pp. 589-604, 2019.

[4] R. Lencioni, "Chemoembolization in patients with hepatocellular carcinoma," Liver Cancer, vol. 1, no. 1, pp. 41-50, 2012.

[5] T. Sattler, C. Bredt, S. Surwald, C. Rust, J. Rieger, and T. Jakobs, "Efficacy and safety of drug eluting bead TACE with microspheres $<150 \mu \mathrm{m}$ for the treatment of hepatocellular carcinoma," Anticancer Research, vol. 38, no. 2, pp. 1025-1032, 2018.

[6] T. K. Rhee, J. Y. Young, A. C. Larson et al., "Effect of transcatheter arterial embolization on levels of hypoxia-inducible factor- $1 \alpha$ in rabbit VX2 liver tumors," Journal of Vascular and Interventional Radiology, vol. 18, no. 5, pp. 639-645, 2007.

[7] K. Liu, X.-L. Min, J. Peng, K. Yang, L. Yang, and X.-M. Zhang, "The changes of HIF- $1 \alpha$ and VEGF expression after TACE in patients with hepatocellular carcinoma," Journal of Clinical Medicine Research, vol. 8, no. 4, pp. 297-302, 2016.

[8] J.-W. Kim, "Hypoxia-inducible factor 1, hepatocellular carcinoma and angiogenesis," The Korean Journal of Hepatology, vol. 16, no. 3, pp. 278-279, 2010.

[9] T. K. Lee, R. T. Poon, A. P. Yuen et al., "Regulation of angiogenesis by Id-1 through hypoxia-inducible factor-1alphamediated vascular endothelial growth factor up-regulation in hepatocellular carcinoma," Clinical Cancer Research, vol. 12, no. 23, pp. 6910-6919, 2016.

[10] Q. Chen, W. Lin, Z. Yin et al., "Melittin inhibits hypoxiainduced vasculogenic mimicry formation and epithelialmesenchymal transition through suppression of HIF-1alpha/ Akt pathway in liver cancer," Evidence-Based Complementary and Alternative Medicine, vol. 2019, Article ID 9602935, 10 pages, 2019.

[11] G. Cane, A. Ginouvès, S. Marchetti et al., "HIF- $1 \alpha$ mediates the induction of IL-8 and VEGF expression on infection with Afa/Dr diffusely adhering E. coli and promotes EMT-like behaviour," Cellular Microbiology, vol. 12, no. 5, pp. 640-653, 2010.

[12] C. X. Dai, Q. Gao, S. J. Qiu et al., "Hypoxia-inducible factor-1 alpha, in association with inflammation, angiogenesis and MYC, is a critical prognostic factor in patients with HCC after surgery," BMC Cancer, vol. 9, p. 418, 2009.

[13] D. Martin, R. Galisteo, and J. S. Gutkind, "CXCL8/IL8 stimulates vascular endothelial growth factor (VEGF) expression and the autocrine activation of VEGFR2 in endothelial cells by activating NF $\kappa \mathrm{B}$ through the CBM (Carma3/ Bcl10/Malt1) complex," Journal of Biological Chemistry, vol. 284, no. 10, pp. 6038-6042, 2009.

[14] R. Lencioni, J. M. Llovet, G. Han et al., "Sorafenib or placebo plus TACE with doxorubicin-eluting beads for intermediate stage HCC: the SPACE trial," Journal of Hepatology, vol. 64, no. 5, pp. 1090-1098, 2016.

[15] M. Kudo, G. Han, R. S. Finn et al., "Brivanib as adjuvant therapy to transarterial chemoembolization in patients with hepatocellular carcinoma: a randomized phase III trial," Hepatology, vol. 60, no. 5, pp. 1697-1707, 2014.

[16] X. Wang, N. Wang, F. Cheung, L. Lao, C. Li, and Y. Feng, "Chinese medicines for prevention and treatment of human hepatocellular carcinoma: current progress on pharmacological actions and mechanisms," Journal of Integrative Medicine, vol. 13, no. 3, pp. 142-164, 2015.
[17] H. Zhao, X. Zhai, Z. Chen et al., "Transarterial chemoembolization combined with Jie-du granule preparation improves the survival outcomes of patients with unresectable hepatocellular carcinoma," Oncotarget, vol. 8, no. 28, pp. 45234-45241, 2017.

[18] C.-q. Ling, J. Fan, H.-s. Lin et al., "Clinical practice guidelines for the treatment of primary liver cancer with integrative traditional Chinese and Western medicine," Journal of Integrative Medicine, vol. 16, no. 4, pp. 236-248, 2018.

[19] L. Y. Chen, X. F. Zhai, Z. Chen et al., "Jie-du granule preparation for the treatment of advanced hepatocellular carcinoma: a retrospective cohort study of 177 patients," Oncotarget, vol. 8, no. 18, pp. 30471-30476, 2017.

[20] Y. Yu, Q. Lang, Z. Chen et al., "The efficacy for unresectable hepatocellular carcinoma may be improved by transcatheter arterial chemoembolization in combination with a traditional Chinese herbal medicine formula," Cancer, vol. 115, no. 22, pp. 5132-5138, 2009.

[21] J. W. Yu, Y. P. Deng, X. Han, G. F. Ren, J. Cai, and G. J. Jiang, "Metformin improves the angiogenic functions of endothelial progenitor cells via activating AMPK/eNOS pathway in diabetic mice," Cardiovascular Diabetology, vol. 15, p. 88, 2016.

[22] S. K. Saha, S. Roy, and A. R. Khuda-Bukhsh, "Ultra-highly diluted plant extracts of Hydrastis canadensis and Marsdenia condurango induce epigenetic modifications and alter gene expression profiles in HeLa cells in vitro," Journal of Integrative Medicine, vol. 13, no. 6, pp. 400-411, 2015.

[23] X.-P. Li, X.-Y. Yang, E. Biskup et al., "Co-expression of CXCL8 and HIF- $1 \alpha$ is associated with metastasis and poor prognosis in hepatocellular carcinoma," Oncotarget, vol. 6, no. 26, pp. 22880-22889, 2015.

[24] J.-L. Raoul, M. Gilabert, and G. Piana, "How to define transarterial chemoembolization failure or refractoriness: a European perspective," Liver Cancer, vol. 3, no. 2, pp. 119-124, 2014.

[25] D. J. Brat, A. C. Bellail, and E. G. Van Meir, "The role of interleukin-8 and its receptors in gliomagenesis and tumoral angiogenesis," Neuro-Oncology, vol. 7, no. 2, pp. 122-133, 2005.

[26] S. Hu, Y. Zhu, X. Xia et al., "Ginsenoside Rg3 prolongs survival of the orthotopic hepatocellular carcinoma model by inducing apoptosis and inhibiting angiogenesis," Analytical Cellular Pathology (Amst), vol. 2019, Article ID 3815786, 7 pages, 2019.

[27] S. S. Kim, H. J. Cho, J. H. Won et al., "Interleukin-8 level as a prognostic marker in patients with hepatitis B virus-associated hepatocellular carcinoma treated with transarterial chemoembolization," Cytokine, vol. 76, no. 2, pp. 449-457, 2015.

[28] R. Sacco, G. Tapete, N. Simonetti et al., "Transarterial chemoembolization for the treatment of hepatocellular carcinoma: a review," Journal of Hepatocellular Carcinoma, vol. 4, pp. 105-110, 2017.

[29] Y. S. Chun, M. S. Kim, and J. W. Park, "Oxygen-dependent and -independent regulation of HIF-1alpha," Journal of Korean Medical Science, vol. 17, no. 5, pp. 581-588, 2002.

[30] W. Cheng, Z. Cheng, Z. Yang, D. Xing, and M. Zhang, "Upregulation of hypoxia-inducible factor lalpha mRNA expression was associated with poor prognosis in patients with hepatocellular carcinoma," OncoTargets and Therapy, vol. 12, pp. 6285-6296, 2019.

[31] R. S. Finn, A. X. Zhu, W. Farah et al., "Therapies for advanced stage hepatocellular carcinoma with macrovascular invasion 
or metastatic disease: a systematic review and meta-analysis," Hepatology, vol. 67, no. 1, pp. 422-435, 2018.

[32] M. J. Kim, J. W. Jang, B. S. Oh et al., "Change in inflammatory cytokine profiles after transarterial chemotherapy in patients with hepatocellular carcinoma," Cytokine, vol. 64, no. 2, pp. 516-522, 2013.

[33] Y. Shen, H. Wang, W. Li, and J. Chen, "Prognostic significance of the CRP/Alb and neutrophil to lymphocyte ratios in hepatocellular carcinoma patients undergoing TACE and RFA," Journal of Clinical Laboratory Analysis, vol. 33, no. 9, p. e22999, 2019.

[34] A. Suner, B. I. Carr, H. Akkiz et al., "Inflammatory markers C-reactive protein and PLR in relation to HCC characteristics," Journal of Translational Science, vol. 5, no. 3, 2019.

[35] Y. Liu, Z. X. Wang, Y. Cao, G. Zhang, W. B. Chen, and C. P. Jiang, "Preoperative inflammation-based markers predict early and late recurrence of hepatocellular carcinoma after curative hepatectomy," Hepatobiliary Pancreat Diseases International, vol. 15, no. 3, pp. 266-274, 2016.

[36] S. H. Loosen, M. Schulze-Hagen, C. Leyh et al., "IL-6 and IL-8 serum levels predict tumor response and overall survival after TACE for primary and secondary hepatic malignancies," International Journal of Molecular Sciences, vol. 19, p. 6, 2018.

[37] X. H. Lu, G. X. Mao, Y. Y. Zhang, Y. S. Chu, H. X. Yuan, and X. Q. Zhu, "Association between variants of IL-8 and IL-10 genes, and efficacy of transcatheter arterial chemoembolization and subsequent prognosis in patients with liver cancer," European Review for Medical and Pharmacological Sciences, vol. 19, no. 17, pp. 3218-3223, 2015.

[38] J. Akiba, H. Yano, S. Ogasawara, K. Higaki, and M. Kojiro, "Expression and function of interleukin-8 in human hepatocellular carcinoma," International Journal of Oncology, vol. 18, no. 2, pp. 257-264, 2001.

[39] W. Feng, T. Xue, S. Huang et al., "HIF-1alpha promotes the migration and invasion of hepatocellular carcinoma cells via the IL-8-NF-kappaB axis," Cellular \& Molecular Biology Letters, vol. 23, p. 26, 2018.

[40] S. H. Choi, O. J. Kwon, J. Y. Park et al., "Inhibition of tumour angiogenesis and growth by small hairpin HIF-1alpha and IL8 in hepatocellular carcinoma," Liver International, vol. 34, no. 4, pp. 632-642, 2014.

[41] N. Li, C. Liu, G. Ma et al., "Asparaginyl endopeptidase may promote liver sinusoidal endothelial cell angiogenesis via PI3K/Akt pathway," Revista Española De Enfermedades Digestivas, vol. 111, no. 3, pp. 214-222, 2019. 